Received Date : 20-Feb-2016

Revised Date : 08-Jun-2016

Accepted Date : 11-Jul-2016

Article type : Short Communication

\title{
Perceived state of self during motion can differentially modulate numerical magnitude allocation
}

\section{Short Communications}

Author affiliations

1. Division of Brain Sciences, Imperial College, Charing Cross Hospital, Fulham Palace Road, London, W6 8RF, UK.

2. Department of Medicina Interna, Sezione di Fisiologia Umana, Universita di Perugia, Perugia, Italy.

3. Department of Experimental Psychology, Oxford University, South Parks Road, Oxford, 0X1 3UD, UK.

Correspondence to be addressed to Dr Qadeer Arshad: q.arshad@imperial.ac.uk

Conflict of interest: the authors report no conflict of interest

Key-words; numerical magnitude, motion perception, spatial attention, vestibular cognition

\section{Behavioural/Cognitive section}

This article has been accepted for publication and undergone full peer review but has not been through the copyediting, typesetting, pagination and proofreading process, which may lead to differences between this version and the Version of Record. Please cite this article as doi: 10.1111/ejn.13335

This article is protected by copyright. All rights reserved. 


\section{ABSTRACT:}

Although a direct relationship between numerical-allocation and spatial-attention has been proposed, recent research suggests these processes are not directly coupled. In keeping with this, spatial attention shifts induced either via visual or vestibular motion can modulate numerical allocation in some circumstances but not in others. In addition to shifting spatial attention, visual or vestibular motion-paradigms also (i) elicit compensatory eye-movements which themselves can influence numerical-processing and (ii) alter the perceptual-state of"self", inducing changes in bodily self-consciousness impacting upon cognitive mechanisms. Thus, the precise mechanism by which motion modulates numerical-allocation remains unknown. We sought to investigate the influence that different perceptual experiences of motion have upon numerical magnitude allocation whilst controlling for both eyemovements and task-related effects. We first used optokinetic visual-motion stimulation (OKS) to elicit the perceptual experience of either "visual world" or "self"-motion during which eye movements were identical. In a second experiment we used a vestibular protocol examining the effects of perceived and subliminal angular rotations in darkness, which also provoked identical eye movements. We observed that during the perceptual experience of "visual-world" motion, rightward OKS biased judgments towards smaller numbers, whereas leftward OKS biased judgments towards larger numbers. During the perceptual experience of "self-motion", judgments were biased towards larger numbers irrespective of the OKS direction. Contrastingly, vestibular motion perception was found not to modulate numerical magnitude allocation, nor was there any differential modulation when comparing "perceived" versus "subliminal" rotations. We provide a novel demonstration that magnitude-allocation can be differentially modulated by the perceptual state of-self during visual-motion.

\section{INTRODUCTION}

The link between numerical allocation of magnitude and spatial attention mechanisms is contentious. On one hand, numerical magnitude is proposed to be superimposed upon a left-to-right spatially orientated representation, termed the mental number line (MNL) (Dehaene et al., 1993). Evidence supporting such an arrangement is provided by the spatial numerical association response code (SNARC) effect (Dehaene et al., 1993), the observation

This article is protected by copyright. All rights reserved. 
that spatial attention shifts occur following number perception and the findings that pathological attentional biases in patients following unilateral brain damage lead to biasing of magnitude estimates towards larger numbers (Zorzi et al., 2002). However, recent studies have demonstrated that number perception does not automatically induce shifts of spatial attention (Fattorini et al., 2015), that a double dissociation exists between physical line and number-pair bisection (Doricchi et al., 2005; Rotondaro et al., 2015) and that there is no relationship between the strength of an attentional shift induced by numbers and the SNARC effect (Fattorini et al., 2015). In addition, work suggesting that there is no inherent right or left side to the MNL (Aiello et al., 2012), and the demonstration of topographical representation of numerosity in the parietal cortex in the absence of any visuo-spatial relationship (Harvey et al., 2013), all provide strong evidence against an intrinsic link between spatial attention and numerical magnitude. Hence, experimental data to date do not converge upon a coherent model of how number and space interact in the human brain.

Regardless of these theoretical issues, there has been increasing scientific interest in the effect of motion upon numerical magnitude perception, which has been explored by activation of either the visual or vestibular sensory systems. Hartmann and colleagues employed whole-body vestibular stimulation to demonstrate a bi-directional relationship between the generation and processing of numerical magnitude and self-motion detection thresholds (Hartmann et al., 2012b). Furthermore, both lateral head turns (Loetscher et al., 2008) and galvanic vestibular stimulation (Ferrè et al., 2013) have been shown to bias the internal 'spontaneous' number generator. A different set of studies have investigated the effects of visual motion upon numerical processing, typically using 'optokinetic' stimulation (OKS). Specifically, in stroke patients with a rightward attentional bias, viewing leftward motion has been shown to temporarily ameliorate the pathological numerical biases that present towards larger numbers during a number pair bisection task (Priftis et al., 2012; Salillas et al., 2009). Further, in normal healthy individuals, a recent study has demonstrated that rightward visual-motion can abolish the classical number size effect during a number comparison task (i.e., faster reaction times for small than large numbers) (Ranzini et al., 2015). In contrast, Salillas and colleagues found that motion direction had no effect in patients with a rightward attentional bias (Salillas et al., 2009). Moreover, we have recently

This article is protected by copyright. All rights reserved. 
shown that perceived self-motion elicited following vestibular stimulation has no effect upon numerical magnitude allocation (Arshad et al., 2016).

Thus, it remains unclear what effect motion perception per se has upon numerical magnitude allocation. We propose that the contradictory findings can be linked to previous studies not controlling for (i) eye movements which can critically influence numerical processing (Loetscher et al., 2010; Ranzini et al., 2015) or (ii) the perceptual state of self during motion (Lopez et al., 2008) impacting upon cognitive mechanisms (Gibbs Jr, 2005) and (iii) the lack of standardisation of the numerical task as these can place differing demands upon both magnitude and visuo-spatial processing resources (Ferrè et al., 2013; Priftis et al., 2006). Accordingly, in the present study we tested the hypothesis that different perceptual states of self during both visual and vestibular mediated motion may differentially modulate numerical magnitude allocation, whilst controlling for both eyemovements and numerical task related effects.

\section{METHODS}

\section{Subjects:}

All Participants were healthy right-handed individuals ( $n=40$ as determined by power calculations, mean age $=21.4$, range 18-29; $22 \mathrm{~F})$ with no history of otological, ophthalmological, psychiatric or neurological disorder. Subjects were randomly allocated into either Group 1 (i.e. visual motion) or 2 (i.e. vestibular motion) ( $n=20 ; 11$ F). All participants were naive to the purpose of the study, and provided written informed consent.

\section{Numerical task:}

Subjects performed a mental number pair bisection task that involved estimating the midpoint between two numbers without calculation (response was required within 3 seconds). Numbers were aurally presented via a radio speaker situated in the midline directly behind the subject. For each test condition the following 20 number pair trials; (33-87), (33-89), (3990), (39-93), (42-67), (44-68), (46-90), (48-92), (54-90), (56-92), (56-92), (59-87), (60-89), (6199), (64-92), (67-95), (50-99), (55-96), (58-124) and (58-132) were presented in a randomised order across the different perceptual conditions (Arshad et al., 2016). Before the experiment began, 3 practice trials were provided to familiarise the subjects with the 
task [(32-56), (41-59), (61-135)]. Bisection errors were calculated by subtracting the true midpoint from the reported midpoint, and percentage bisection errors were calculated by dividing the errors by the number pair interval size. Positive \% bisection errors denoted overestimations, whereas negative \% bisection errors denoted underestimation from the true midpoint (Arshad et al., 2016).

\section{Experiment 1: Visually mediated changes in self-motion perception}

Subjects were seated on a motorised rotating chair surrounded by a visual stimulus that consisted of a $1.44 \mathrm{~m}$ diameter drum marked with vertical black and white stripes. Subjects viewed the drum at a fixed distance of 0.72 metres (Figure 1). To generate the OKS, the drum was rotated around the subject in an Earth-vertical axis at a constant velocity of $40 \%$ second, either right or leftwards (randomised order) (Arshad et al., 2014). Viewing fullfield OKS as implemented herein, can induce two different possible perceptual states: the perception of either "visual world" or "self"-motion (Kleinschmidt et al., 2002; Thilo et al., 2000) (Figure $1 A \& B$ ). The initial percept is that of visual world motion and typically after 20 seconds of viewing full-field OKS, the subjective experience transitions to that of selfmotion. Once in this perceptual state, subjects typically continue to experience self-motion until the OKS ceases. Accordingly, for the "self-motion" condition, we waited for 30 seconds after the onset of visual motion and verbally confirmed with the subject the perceptual state but not the perceived direction experienced (i.e. avoiding spatial labelling confounds) before proceeding with the numerical task. To elicit the perceptual state of "world-motion" during OKS, subjects were asked to remove their feet from the suspended foot rest attached to the rotating chair and plant them on the floor, so as to provide a strong proprioceptive cue that they were stationary (Figure 1B). Our pilot work demonstrated that the deployment of this strategy successfully negated the sensation of vection in all subjects. Subjects viewed the OKS stimulus moving for 30 seconds during the world-motion condition before the commencement of the numerical task (i.e. matched time-delay). Subjects performed number-pair bisection task under three conditions: (1) no OKS (i.e. baseline), (2) viewing OKS either right or leftward (randomised order) that induced the perceptual experience of world-motion and (3) viewing OKS either right or leftwards during the perceptual experience of self-motion.

This article is protected by copyright. All rights reserved. 


\section{Experiment 2: Vestibular mediated changes in self-motion perception}

Subjects were seated on a vibration free motorised rotating chair in darkness (Contraves, USA). For perceived rotations, either right or leftwards (randomised order), a symmetrical sinusoidal rotation profile was employed. The sinusoidal rotation profile had an amplitude of $40^{\circ}$ and a velocity of $11^{\circ} / \mathrm{s}$ (Figure 2). For subliminal rotations (either right or leftwards), we implemented an asymmetrical sinusoidal rotation profile, with an amplitude of $40^{\circ}$, but two separate velocity components, a fast phase with a velocity of $50 \%$ and a slow phase with a velocity of $11 \%$ (Figure 2) (Pettorossi et al., 2013). This ensured that the slow phase component of the asymmetrical rotation was identical to the velocity employed in the symmetrical rotation. The perceptual experience of this asymmetrical rotation paradigm was that after 30 seconds of rotation, subjects only perceive the fast and not the slow phase component. Importantly, the vestibular nystagmus elicited during the slow phase component of the subliminal rotations is identical to that observed during perceived rotations (Pettorossi et al., 2013). Subjects were instructed not to pay attention to the direction of rotation and rather focus fully on the numerical task. Subjects performed number-pair bisection during (1) no motion (i.e. baseline measure), and 30 seconds after the onset of chair motion (i.e. matched time delay to experiment 1) during either (2) perceived right or leftward rotations (symmetrical rotation profile) and (3) subliminal right or leftward rotations (asymmetrical rotation profile).

\section{Eye movement analysis and signal recordings:}

Eye movements were recorded using bi-temporal electro-oculography (EOG). Subjects initially performed a calibration of the eye signal with a $20^{\circ}$ saccadic target either to the right or left of centre. After the calibration of the eye signal all subsequent file recordings including those of the calibration were saved at a sampling rate of $250 \mathrm{~Hz}$. Eye movement analysis was performed offline using in-house software programme; Analysis (author; $\mathrm{Mr}$ David Buckwell). OKS drum velocity (experiment 1) and chair position signals (experiment 2) were additionally recorded at $250 \mathrm{~Hz}$.

\section{RESULTS}

\section{Experiment 1: Visually mediated changes in self-motion perception}

Firstly we assessed for differences in all perceptual conditions versus baseline measures (i.e. right and leftward visual world and right and leftward self-motion). All were found to be 
significant ( $p<0.05$; paired t-test; Bonferroni corrected). Accordingly, for both further statistical and illustrative purposes (Fig 3), we subtracted the baseline from each condition and performed a 2x2 Repeated measures ANOVA, with factors direction ( 2 levels: right; left) $x$ perceptual condition ( 2 levels: world motion; self-motion). This revealed a significant main effect for perceptual condition $(F(1,19)=34.2, P<0.0001)$, main effect for direction $(F(1,19)=$ 22.1, $\mathrm{p}<0.0001)$, and a significant interaction for direction*perceptual condition $(F(1,19)=27.2, p<0.0001)$. Post-hoc paired t-tests with Bonferroni corrections revealed significant differences for the following conditions (rightward visual world vs leftward visual world; $p<0.001$; rightward visual world vs rightward self-motion; $p<0.001$ and rightward visual world vs leftward self-motion $p<0.001$. However there was no significant difference between leftward visual world vs leftward self-motion; $p>0.05$. For the statistical analysis of the eye movements (i.e. OKN gain) associated with OKS, a $2 \times 2$ repeated measures ANOVA with factors: direction ( 2 levels: right; left) and perceptual condition ( 2 levels: world motion; self-motion), revealed no significant main effect for the gain of the eye movement for neither direction $(F(1,19)=1.41, P>0.05)$ nor perceptual condition $F(1,19)=1.22, P>0.05)$ and no interaction $(F(1,19)=1.11, P>0.05)$.

\section{Experiment 2: Vestibular mediated changes in self-motion perception}

Firstly we assessed for differences in all perceptual conditions versus baseline measures (i.e. right and leftward perceived rotations and right and leftward subliminal rotations). All were found to be non-significant ( $p>0.05$ ) paired t-test; Bonferroni corrected. Accordingly, for both further statistical and illustrative purposes (Fig 4), we subtracted the baseline from each condition and performed a $2 \times 2$ Repeated measures ANOVA, with factors direction (2 levels: right; left) x perceptual condition (2 levels: perceived motion; subliminal motion). This revealed no significant main effect for neither direction $(F(1,19)=0.822, p>0.05)$, nor perceptual condition $(F(1,19)=0.76, p>0.05)$ and no interaction $(F(1,19)=0.654, P>0.05)$. For the statistical analysis of the eye movement (i.e. gain of the vestibular nystagmus),a $2 \times 2$ repeated measures ANOVA, with factors: direction (2 levels: right; left) and perceptual condition (2 levels: perceived motion; subliminal motion) revealed no significant main effect for the gain of vestibular nystagmus for neither direction $(F(1,19)=0.482, p>0.05)$ nor perceptual condition $(F(1,19)=1.34, p>0.05)$ and no interaction $(F(1,19)=0.919, P>0.05)$.

This article is protected by copyright. All rights reserved. 


\section{DISCUSSION}

Our findings provide the first demonstration that the allocation of numerical magnitude can be differentially modulated by the perceptual state of self that is experienced during visual but not vestibular mediated motion. Notably, our experimental design controlled for both any task-associated variability (Priftis et al., 2006) or eye-movement related effects (Ranzini et al., 2015) upon numerical processing.

During visual motion that elicited the perceptual experience of world-motion, rightward OKS, that elicits left-beating OKN, shifted spatial attention leftwards (Teramoto et al., 2004; Watanabe, 2001), biasing judgments towards smaller numbers. Conversely, leftward OKS, that elicits right beating OKN, shifted spatial attention rightwards (Teramoto et al., 2004; Watanabe, 2001), biasing judgments towards larger numbers. Thus, during world-motion perception, numerical biasing occurred as result of eye-movement related spatial attention shifts in a manner consistent with context-dependent encoding of small numbers in association with the left side of space through disproportionate representation in the right hemisphere, with larger numbers being represented in association with rightward space in the left hemisphere (Arshad et al., 2016). Contrastingly, during visual motion that elicited the perceptual experience of self-motion (i.e. vection), both right and leftward motion biased numerical judgments towards larger numbers. Here, neither eye-movements nor any related spatial attention biases can explain the observed numerical modulation (Ranzini et al., 2015) as both the eye movements and secondary spatial attention shifts were in opposing directions during left and rightward OKS, yet judgments were consistently biased towards larger magnitudes.

Previous neuroimaging data have demonstrated that during vection, conflict occurs between visual and vestibular cortical areas, resulting in inhibition of vestibular cortical areas (Brandt et al., 1998). Cortical areas devoted to vestibular processing overlap considerably with the right-lateralised fronto-parietal attentional network with both appearing to have right hemispheric dominance (Corbetta and Shulman, 2002; Dieterich et al., 2003; Lopez et al., 2012; Zu Eulenburg et al., 2012). Accordingly, we propose that during vection, inhibition of vestibular cortical areas (Brandt et al., 1998), preferentially in the right

This article is protected by copyright. All rights reserved. 
hemisphere (Arshad et al., 2015a; Arshad et al., 2015b; Arshad et al., 2013; Dieterich et al., 2003), results in a left hemisphere predominant response, biasing subjects towards larger numerical magnitudes (Arshad et al., 2016). This account is line with both (i) the previous observations of a pathological bias towards larger numbers during large-interval number pair bisection following right hemisphere lesions (Zorzi et al., 2002) which was found to be disassociated from spatial neglect (Doricchi et al., 2005) and (ii) our recent findings, using a combined visuo-vestibular stimulation paradigm that can result in a left-hemisphere predominant response which biases judgments towards larger magnitudes (Arshad et al., 2016). Hence, numerical biasing towards larger magnitudes during the perceptual experience of self-motion during OKS occurred via a hemispheric-mediated effect, and the fact that this results in the dissociation of numerical-spatial interactions is in line with previous findings from both brain damaged and healthy individuals (Aiello et al., 2012; Arshad et al., 2016).

Contrastingly, we found that vestibular-mediated motion perception did not modulate numerical magnitude. That is, perceived angular rotations did not bias numerical judgments in comparison to measures obtained at either baseline (i.e. no motion) or to those obtained during subliminal angular rotations. Previously it has been demonstrated that vestibular activation elicited via either linear translation (Hartmann et al., 2012a), lateral head turns (Loetscher et al., 2008) or galvanic stimulation (Ferrè et al., 2013) can modulate numerical cognition. We propose the fact that we did not observe any modulation of magnitude allocation during vestibular activation can be attributed to the numerical task employed in our present study compared to those which have been previously employed, as different numerical-tasks place differing demands upon both magnitude and visuo-spatial processing resources (Priftis et al., 2006). Further, the lack of any modulatory effect during angular vestibular rotations upon numerical magnitude is in line with our recent findings that demonstrated no effect upon number-pair bisection during caloric vestibular stimulation (Arshad et al., 2016).

To conclude we provide the first demonstration that numerical magnitude can be differentially modulated dependent upon the perceptual state of self (i.e. changes in the sense of embodiment and/or bodily self-consciousness) during visual but not vestibular mediated motion.

This article is protected by copyright. All rights reserved. 


\section{REFERENCES}

Aiello, M., Jacquin-Courtois, S., Merola, S., Ottaviani, T., Tomaiuolo, F., Bueti, D., Rossetti, Y. \& Doricchi, F. (2012) No inherent left and right side in human 'mental number line': Evidence from right brain damage. Brain, 135, 2492-2505.

Arshad, Q., Patel, M., Goga, U., Nigmatullina, Y. \& Bronstein, A. (2015a) Role of handedness-related vestibular cortical dominance upon the vestibular-ocular reflex. J. Neurol., 262, 1069-1071.

Arshad, Q., Siddiqui, S., Ramachandran, S., Goga, U., Bonsu, A., Patel, M., Roberts, R., Nigmatullina, Y., Malhotra, P. \& Bronstein, A. (2015b) Right hemisphere dominance directly predicts both baseline V1 cortical excitability and the degree of top-down modulation exerted over low-level brain structures. Neuroscience, 311, 484-489.

Arshad, Q., Nigmatullina, Y. \& Bronstein, A. (2014) Unidirectional visual motion adaptation induces reciprocal inhibition of human early visual cortex excitability. Clinical Neurophysiology, 125, 798-804.

Arshad, Q. \& Bronstein, A. Bidirectional modulation of numerical magnitude.

Arshad, Q., Nigmatullina, Y. \& Bronstein, A.M. (2013) Handedness-related cortical modulation of the vestibular-ocular reflex. J. Neurosci., 33, 3221-3227.

Brandt, T., Bartenstein, P., Janek, A. \& Dieterich, M. (1998) Reciprocal inhibitory visual-vestibular interaction. visual motion stimulation deactivates the parieto-insular vestibular cortex. Brain, 121 ( Pt 9), 1749-1758.

Corbetta, M. \& Shulman, G.L. (2002) Control of goal-directed and stimulus-driven attention in the brain. Nature reviews neuroscience, 3, 201-215.

Dehaene, S., Bossini, S. \& Giraux, P. (1993) The mental representation of parity and number magnitude. J. Exp. Psychol. : Gen., 122, 371.

Dieterich, M., Bense, S., Lutz, S., Drzezga, A., Stephan, T., Bartenstein, P. \& Brandt, T. (2003) Dominance for vestibular cortical function in the non-dominant hemisphere. Cereb. Cortex, 13, 994-1007.

Doricchi, F., Guariglia, P., Gasparini, M. \& Tomaiuolo, F. (2005) Dissociation between physical and mental number line bisection in right hemisphere brain damage. Nat. Neurosci., 8, 1663-1665.

Fattorini, E., Pinto, M., Rotondaro, F. \& Doricchi, F. (2015) Perceiving numbers does not cause automatic shifts of spatial attention. Cortex, 73, 298-316.

Ferrè, E. R., Vagnoni, E. \& Haggard, P. (2013) Galvanic vestibular stimulation influences randomness of number generation. Experimental brain research, 224, 233-241.

Gibbs Jr, R. W. (2005) Embodiment and Cognitive Science. Cambridge University Press, .

Hartmann, M., Grabherr, L. \& Mast, F.W. (2012a) Moving along the mental number line: Interactions between whole-body motion and numerical cognition. Journal of Experimental Psychology: Human Perception and Performance, 38, 1416.

Hartmann, M., Farkas, R. \& Mast, F.W. (2012b) Self-motion perception influences number processing: Evidence from a parity task. Cognitive Processing, 13, S189-92.

This article is protected by copyright. All rights reserved. 
Harvey, B. M., Klein, B.P., Petridou, N. \& Dumoulin, S.O. (2013) Topographic representation of numerosity in the human parietal cortex. Science, 341, 1123-1126.

Kleinschmidt, A., Thilo, K.V., Buchel, C., Gresty, M.A., Bronstein, A.M. \& Frackowiak, R.S. (2002) Neural correlates of visual-motion perception as object- or self-motion. Neuroimage, 16, 873-882.

Loetscher, T., Bockisch, C.J., Nicholls, M.E. \& Brugger, P. (2010) Eye position predicts what number you have in mind. Current Biology, 20, R264-R265.

Loetscher, T., Schwarz, U., Schubiger, M. \& Brugger, P. (2008) Head turns bias the brain's internal random generator. Current Biology, 18, R60-R62.

Lopez, C., Blanke, O. \& Mast, F. (2012) The human vestibular cortex revealed by coordinate-based activation likelihood estimation meta-analysis. Neuroscience, 212, 159-179.

Lopez, C., Halje, P. \& Blanke, O. (2008) Body ownership and embodiment: Vestibular and multisensory mechanisms. Neurophysiologie Clinique/Clinical Neurophysiology, 38, 149-161.

Pettorossi, V. E., Panichi, R., Botti, F.M., Kyriakareli, A., Ferraresi, A., Faralli, M., Schieppati, M. \& Bronstein, A.M. (2013) Prolonged asymmetric vestibular stimulation induces opposite, long-term effects on self-motion perception and ocular responses. J. Physiol. (Lond. ), 591, 1907-1920.

Priftis, K., Zorzi, M., Meneghello, F., Marenzi, R. \& Umiltà, C. (2006) Explicit versus implicit processing of representational space in neglect: Dissociations in accessing the mental number line. J. Cogn. Neurosci., 18, 680-688.

Priftis, K., Pitteri, M., Meneghello, F., Umilta, C. \& Zorzi, M. (2012) Optokinetic stimulation modulates neglect for the number space: Evidence from mental number interval bisection. Front. Hum. Neurosci., 6, 23.

Ranzini, M., Lisi, M., Blini, E., Pitteri, M., Treccani, B., Priftis, K. \& Zorzi, M. (2015) Larger, smaller, odd or even? task-specific effects of optokinetic stimulation on the mental number space. Journal of cognitive psychology, 27, 459-470.

Rotondaro, F., Merola, S., Aiello, M., Pinto, M. \& Doricchi, F. (2015) Dissociation between line bisection and mental-number-line bisection in healthy adults. Neuropsychologia, 75, 565-576.

Salillas, E., Granà, A., Juncadella, M., Rico, I. \& Semenza, C. (2009) Leftward motion restores number space in neglect. Cortex, 45, 730-737.

Teramoto, W., Watanabe, H., Umemura, H., Matsuoka, K. \& Shinichi, K. (2004) Judgment biases of temporal order during apparent self-motion. IEICE Trans. Inf. Syst., 87, 1466-1476.

Thilo, K. V., Guerraz, M., Bronstein, A.M. \& Gresty, M.A. (2000) Changes in horizontal oculomotor behaviour coincide with a shift in visual motion perception. Neuroreport, 11, 1987-1990.

Watanabe, K. (2001) Modulation of spatial attention with unidirectional field motion: An implication for the shift of the OKN beating field. Vision Res., 41, 801-814.

Zorzi, M., Priftis, K. \& Umiltà, C. (2002) Brain damage: Neglect disrupts the mental number line. Nature, 417, 138-139.

Zu Eulenburg, P., Caspers, S., Roski, C. \& Eickhoff, S.B. (2012) Meta-analytical definition and functional connectivity of the human vestibular cortex. Neuroimage, 60, 162-169.

This article is protected by copyright. All rights reserved. 


\section{Legends}

Figure 1

Visual stimulation paradigm; (A) For the perceived self-motion condition subjects were seated in the motorised Barany chair whilst surrounded by the vertical black and white stripes of the opto-kinetic drum, which was rotated at a velocity of $40^{\circ} / \mathrm{sec}$, either rightwards (red filled arrow) or leftwards (blue filled arrow). Note that the subjects feet were enclosed in the suspended foot rest attached to the rotating chair. Rightward OKS (red filled arrow) induces a perceived self-rotation to the left (as indicated by the red and white checkerboard filled arrow), whereas leftward OKS (blue filled arrow) induces a perceived self-rotation to the right (as indicated by the blue and white checkerboard filled arrow). (B) For the visual world motion condition, exactly the same experimental set up was implemented apart from the fact that now the subject's feet were placed firmly on the ground as to provide a proprioceptive cue to negate the sense of vection (i.e. self-motion). Rightward OKS (as indicated by the red filled arrow) induced a percept of world visual motion to the right, whereas leftward OKS (as indicated by the blue filled arrow) induced a percept of world visual motion to the left. In both conditions (A \& B) eye movements were recorded using bi-temporal EOG electrodes.

\section{Figure 2}

Vestibular stimulation paradigm; (A) Subjects were seated on a vibration free motorised Barany chair, which rotated (angular) either rightwards (red arrow) or leftwards (blue arrow). Eye movements were recorded using bi-temporal EOG electrodes. (B) Velocity and position profiles of rotations employed. For perceived rotations (upper panel) a symmetrical velocity profile was implemented with an amplitude of 40 degrees and a velocity of $11^{\circ} / \mathrm{sec}$ for both right and leftward rotations. In this condition, subjects perceived both directions of rotation. For subliminal rotations an asymmetrical rotation profile was implemented (lower panel). As shown, the sinusoidal rotations also had amplitude of 40 degrees; however the velocity profile was different. That is, there were two components, a slow phase component with a velocity of $11 \% \mathrm{sec}$ (leftwards in the figure) and a fast phase component with a velocity of $50 \% \mathrm{sec}$ (rightwards in the figure). The perceptual consequence of this velocity 
profile was that during rotation subjects only perceived the fast phase of the rotation and not the slow phase component.

\section{Figure 3}

Summary of the results for the visually mediated changes in motion perception experiment; Bisection \% error change from the baseline is represented on the $Y$ axis. On the $X$ axis we represent the different perceptual conditions. For illustrative purposes we have subtracted out the baseline from each condition, hence assuming baseline is at 0 . The line in the middle of the box plot represent the median bisection error. The upper and lower boundaries of the box represent the $25^{\text {th }}$ and $75^{\text {th }}$ percentile. The whiskers represent the $10^{\text {th }}$ and $90^{\text {th }}$ percentile. During the perceptual state of visual world motion, rightward OKS biased subjects towards negative bisection errors (i.e. smaller magnitudes), whereas during leftward OKS subjects were biased towards larger numbers. During the perceptual state of self-motion, both right and leftward OKS biased subjects towards larger numbers. Data marked $^{* *}$ is significant at $p<0.01$. The inserts represent each individual subject's $\%$ change in bisection error relative to the rightward visual world perceptual condition.

\section{Figure 4}

Summary of the results for the vestibular mediated changes in self-motion perception experiment; Bisection \% error change relative to the baseline is represented on the $\mathrm{Y}$ axis. On the $X$ axis we represent the different perceptual conditions. For illustrative purposes we have subtracted out the baseline from each condition, hence baseline is it 0 . The line in the middle of the box plot represent the median bisection error. The upper and lower boundaries of the box represent the $25^{\text {th }}$ and $75^{\text {th }}$ percentile. The whiskers represent the $10^{\text {th }}$ and $90^{\text {th }}$ percentile. No significant differences were observed between any of 4 perceptual conditions during either perceived or subliminal rotations relative to each other or the baseline measure respectively.

This article is protected by copyright. All rights reserved. 
Figure 1

Figure 1

A: Self-motion condition

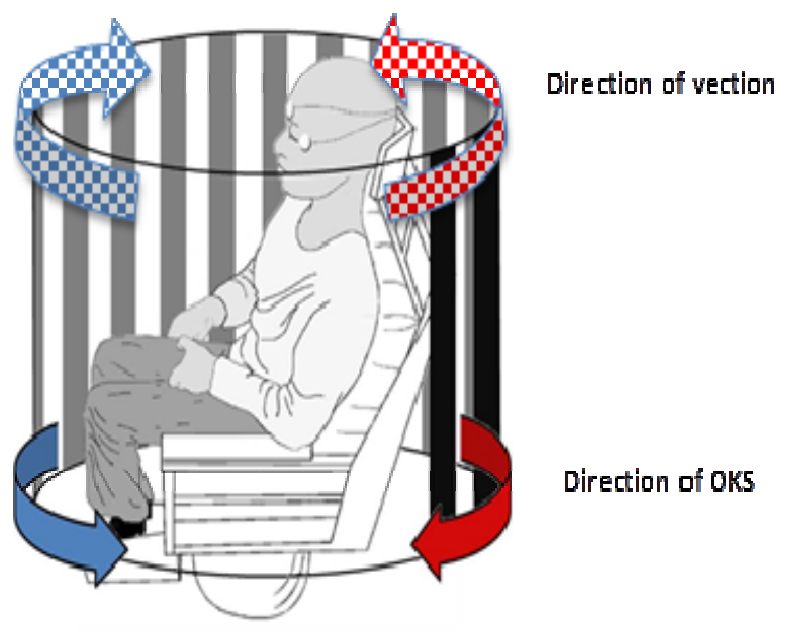

B: World-motion condition

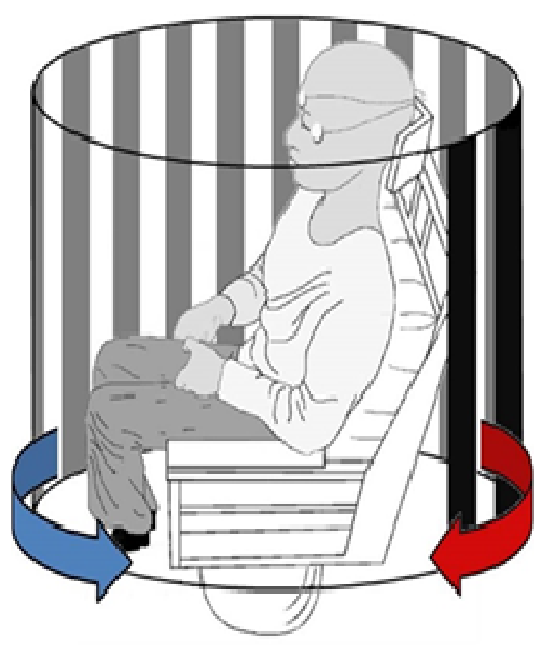


Figure 2
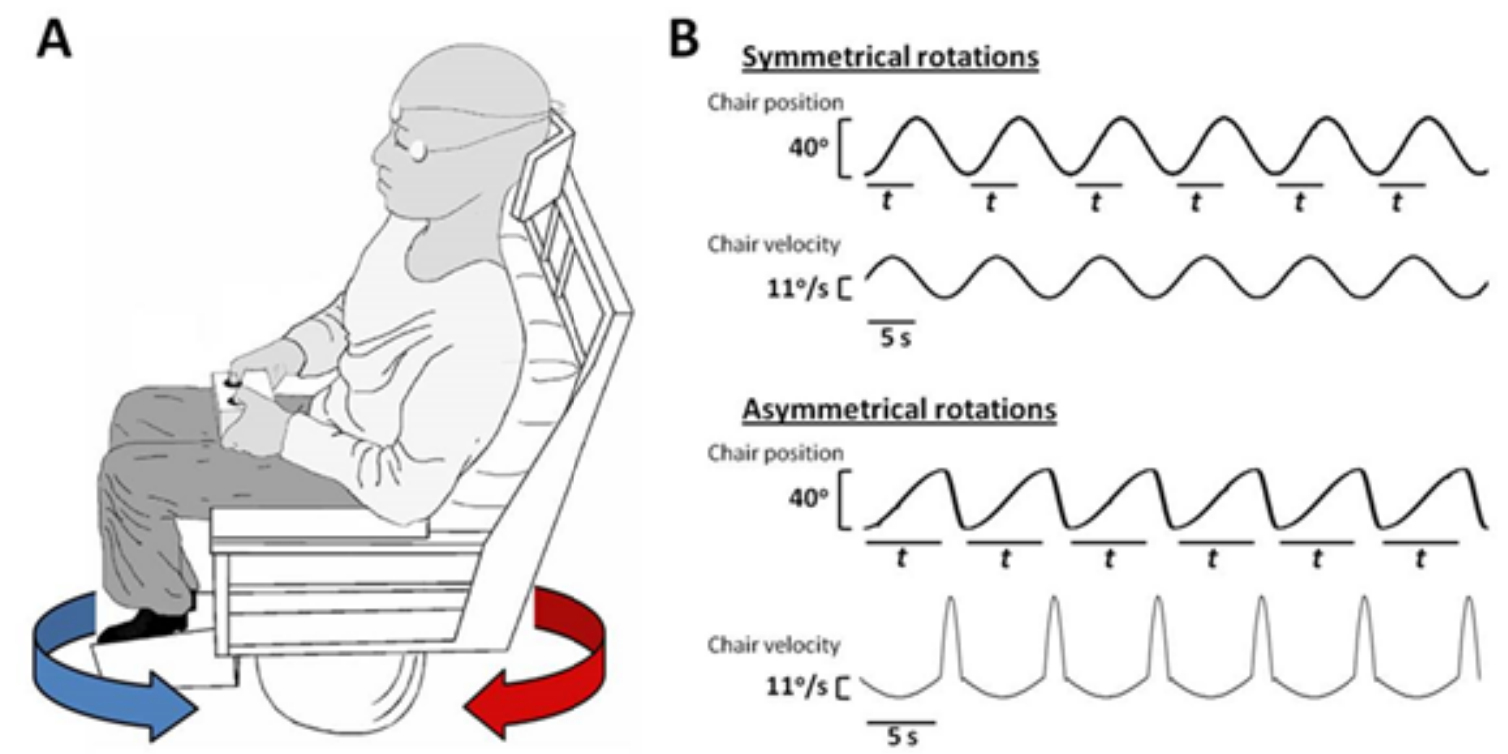

\section{Asymmetrical rotations}

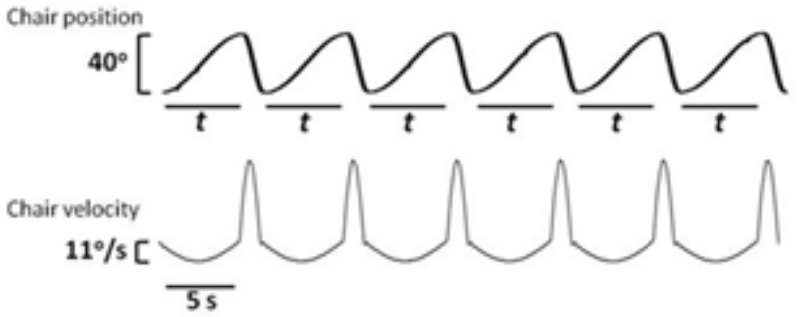

This article is protected by copyright. All rights reserved. 

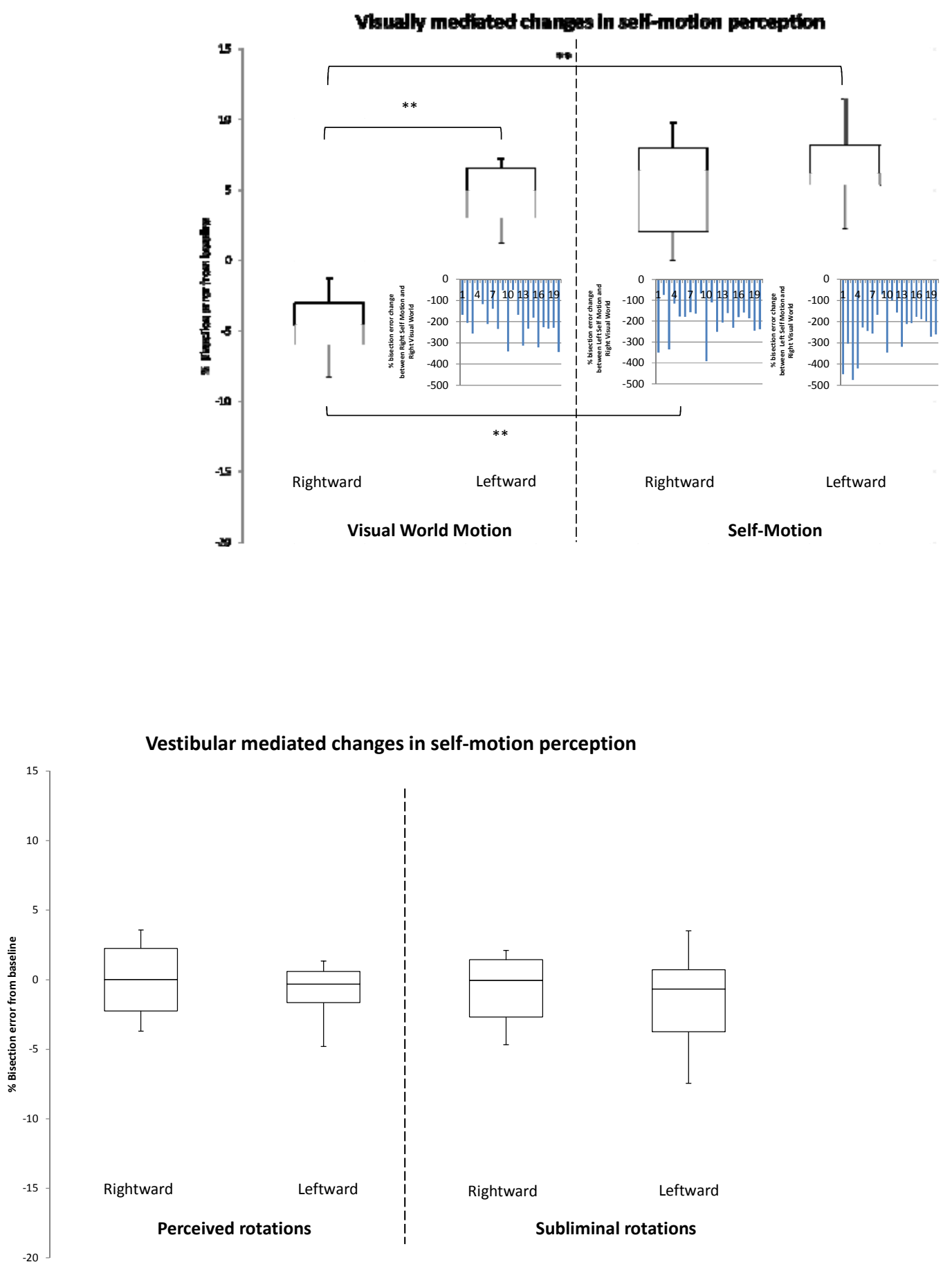

This article is protected by copyright. All rights reserved. 